\title{
Fatores associados a humanização do parto em uma maternidade do Sul do Estado
}

\section{do Maranhão}

\author{
Factors associated with the humanization of childbirth in a maternity hospital in the south of the \\ state of Maranhão
}

Factores asociados con la humanización del parto en una maternidad del sur del estado de

\section{Maranhão}

Recebido: 25/04/2021 | Revisado: 04/05/2021 | Aceito: 11/05/2021 | Publicado: 27/05/2021

\author{
Phablo de Oliveira Souza \\ ORCID: https://orcid.org/0000-0001-5579-5072 \\ Universidade Federal do Maranhão, Brasil \\ E-mail: pablo_sousa_d3@hotmail.com \\ Volmar Morais Fontoura \\ ORCID: https://orcid.org/0000-0001-8731-6750 \\ Universidade Estadual do Tocantins, Brasil \\ E-mail: volmar_morais@hotmail.com \\ Floriacy Stabnow Santos \\ ORCID: https://orcid.org/0000-0001-9875-2283 \\ Universidade Federal do Maranhão, Brasil \\ E-mail: floriacys@gmail.com \\ Marcelino Santos Neto \\ ORCID: https://orcid.org/0000-0002-6105-1886 \\ Universidade Federal do Maranhão, Brasil \\ E-mail: marcelinosn@gmail.com \\ Marcela de Oliveira Feitosa \\ ORCID: https://orcid.org/0000-0003-3017-2922 \\ Universidade Federal do Maranhão, Brasil \\ E-mail: marcelafeitosa_cz@hotmail.com \\ Jhennyfer Barbosa de Oliveira Mantesso \\ ORCID: https://orcid.org/0000-0002-8821-6716 \\ Universidade Federal do Maranhão, Brasil \\ E-mail: jhennyfer.barbosa@ufma.br \\ Maikon Graepp Fontoura \\ ORCID: https://orcid.org/0000-0001-5514-4114 \\ Centro Universitário do Maranhão, Brasil \\ E-mail: maikon2.0@ hotmail.com \\ Iolanda Graepp-Fontoura \\ ORCID: https://orcid.org/0000-0002-9201-480X \\ Universidade Federal do Maranhão, Brasil \\ E-mail: iolanda.graepp@ufma.br
}

\begin{abstract}
Resumo
O Parto Humanizado consiste em um conjunto de estratégias que são adotadas pela equipe de enfermagem junto aos demais profissionais que visa proporcionar o processo parturitivo mais confortável e menos doloroso a mulher. Identificar fatores associados à humanização da assistência durante o trabalho de parto, parto e nascimento. Trata-se de um estudo transversal de caráter descritivo - exploratório e de abordagem quantitativa realizada no Hospital Regional Materno Infantil de Imperatriz (HRMI) no período de outubro a dezembro de 2019. A pesquisa contou com a participação de 120 puérperas de parto vaginal com idades entre 18 e 41 anos. As participantes eram em sua maioria de cor parda e com renda de até 1 salário-mínimo. A grande maioria considerou que houve humanização no seu parto por meio das condutas adotadas pela equipe. Com relação a aplicação dos métodos não farmacológicos de alívio da dor na hora do parto, mais de $60 \%$ das participantes informaram terem recebido esse tipo de assistência, o que indica a adoção de práticas humanizadas. Constatou-se nessa pesquisa boas práticas de atenção assistencial no parto de acordo com a percepção das puérperas. Os profissionais promoviam ações com objetivo de tornar o trabalho de parto e parto um processo mais eficaz e menos doloroso. As condutas realizadas pela equipe de enfermagem e mostradas nesse estudo são princípios para a adoção do parto humanizado, o que proporciona a mulher um parto mais seguro e humano contribuindo para o bem-estar materno-infantil.
\end{abstract}

Palavras-chave: Parto; Humanização; Assistência de enfermagem; Parturiente. 


\begin{abstract}
Humanized Childbirth consists of a set of strategies that are adopted by the nursing team with other professionals that aims to provide the most comfortable and less painful parturition process for women. To identify factors associated with the humanization of care during labor, delivery and birth. This is a cross-sectional study of descriptive exploratory and quantitative approach carried out at the Regional Maternal And Child Hospital of Imperatriz (HRMI) from October to December 2019. The research had the participation of 120 puerperal women of vaginal delivery aged between 18 and 41 years. The participants were mostly brown and with an income of up to 1 minimum wage. The vast majority considered that there was humanization in their delivery through the conducts adopted by the team. Regarding the application of non-pharmacological pain relief methods at the time of delivery, more than $60 \%$ of the participants reported having received this type of care, which indicates the adoption of humanized practices. Good practices of care in childbirth were found in this research according to the perception of postpartum women. The professionals promoted actions with the objective of making labor and delivery a more effective and less painful process. The conducts performed by the nursing team and shown in this study are principles for the adoption of humanized delivery, which provides women with a safer and more humane delivery contributing to maternal and child well-being.
\end{abstract}

Keywords: Parturition; Humanization; Nursing care; Mother.

\title{
Resumen
}

El parto humanizado consiste en un conjunto de estrategias que son adoptadas por el equipo de enfermería con otros profesionales que tiene como objetivo proporcionar el proceso de parturición más cómodo y menos doloroso para las mujeres. Identificar los factores asociados con la humanización de la atención durante el parto, el parto y el nacimiento. Se trata de un estudio transversal de enfoque descriptivo - exploratorio y cuantitativo llevado a cabo en el Hospital Regional Materno-Infantil de Imperatriz (HRMI) de octubre a diciembre de 2019. La investigación contó con la participación de 120 mujeres puerperales de parto vaginal de entre 18 y 41 años. Los participantes eran en su mayoría marrones y con un ingreso de hasta 1 salario mínimo. La gran mayoría consideró que había humanización en su entrega a través de las conductas adoptadas por el equipo. En cuanto a la aplicación de métodos no farmacológicos de alivio del dolor en el momento del parto, más del 60\% de los participantes informaron haber recibido este tipo de atención, lo que indica la adopción de prácticas humanizadas. En esta investigación se encontraron buenas prácticas de atención en el parto de acuerdo con la percepción de las mujeres posparto. Los profesionales promovieron acciones con el objetivo de hacer del trabajo y la entrega un proceso más eficaz y menos doloroso. Las conductas realizadas por el equipo de enfermería y mostradas en este estudio son principios para la adopción del parto humanizado, que proporciona a las mujeres un parto más seguro y humano que contribuye al bienestar materno infantil.

Palabras clave: Parto; Humanización; Cuidado de enfermeira; Madre.

\section{Introdução}

A gestação é uma fase importante na vida de qualquer mulher e corresponde ao período que antecede o parto. É uma fase de mudanças físicas acompanhadas de alterações emocionais e um período em que a mulher pode ficar mais vulnerável (Marins et. al., 2020). O parto cesariano é muito abordado em países desenvolvidos e em desenvolvimento, em especial os asiáticos (sendo mais de 50\% na China), enquanto no Irã a taxa de cesarianas é superior 15\%, muito acima do que a esperada pela Organização Mundial da Saúde (OMS) (Siabani, 2019).

A Organização Mundial da Saúde (OMS) define o parto humanizado como uma série de cuidados planejados e prestados as mulheres visando a integridade de sua dignidade, privacidade e confidencialidade garantindo a liberdade de danos e maus tratos, o que permite a escolha no parto e apoio contínuo durante o trabalho de parto (Mselle; Kohi; Dol, 2018). O Brasil é um dos países em que apresenta as maiores taxas de cirurgias cesarianas em todo mundo, em torno de 55,6\% e apresenta certa diferença quanto a forma de financiamento, em instituições públicas são em torno de $40 \%$ e na rede privada em 85\% (Marian et. al., 2018).

O Ministério da Saúde propõe a humanização no parto visando assegurar uma boa assistência pré-natal realizada tanto por médicos e enfermeiros, como também o incentivo a gestante ao parto normal, uma vez que, quando a gestante é bem acompanhada pela equipe de saúde durante toda sua gestação, o parto vaginal oferece menos riscos e uma rápida recuperação (Brasil, 2002). A atenção humanizada no parto refere-se à necessidade de um olhar diferenciado, compreendendo-o como uma experiência verdadeiramente humana. Dessa forma, acolher, ouvir, orientar e criar vínculos são aspectos extremamente 
fundamentais no cuidado a mulher no processo parturitivo (Possati et. al., 2017).

Estudos mostram que existem práticas úteis e que devem ser usadas em mulheres em trabalho de parto normal, como a liberdade de movimentação, o uso de métodos não farmacológicos de alívio da dor, deambulação e o uso do partograma, que, além de serem estratégias não invasivas são práticas de parto humanizado (Sousa et. al., 2016). O uso de métodos não farmacológicos para alívio da dor como a hidroterapia, exercícios respiratórios, massagens e mudanças de posição faz com que ocorra a substituição de anestésicos e/ou analgésicos durante o trabalho de parto, e o processo seja o mais natural possível (Camargo et. al., 2019).

Para o bom desenvolvimento do trabalho de parto é necessário o bem-estar físico e emocional da mulher, o que reduz os riscos e complicações. Para tanto, o respeito ao direito da mulher a privacidade, a segurança, a assistência humana e de qualidade, aliado ao apoio familiar durante a parturição, transformam o nascimento em um momento único e especial, além de reduzirem os riscos de morbidade e mortalidade (Pereira et. al., 2016).

O parto humanizado ainda é uma prática pouca abordada nas maternidades, mesmo com protocolos vigentes do Ministério da Saúde, uma vez que, ainda persiste a ideia de o parto ser de forma invasiva e medicalizada indo contra o fisiológico do corpo da mulher. Com isso, os profissionais envolvidos nesse processo não oferecem a assistência adequada e tampouco o apoio necessário a necessidade de cada mulher. Dessa forma, destaca-se a importância do parto humanizado que trabalha sob uma perspectiva menos invasiva, medicalizada e hospitalar. Considerando o grau de relevância sobre esta temática, o estudo teve como objetivo identificar fatores associados à humanização da assistência durante o trabalho de parto, parto e nascimento.

\section{Metodologia}

Trata-se de um estudo transversal de caráter descritivo - exploratório e de abordagem quantitativa (Estrela,2018). Foi realizado no Hospital Regional Materno Infantil de Imperatriz, Maranhão, Brasil no período de outubro a dezembro de 2019. A coleta de dados se deu por meio da aplicação de um formulário previamente elaborado contendo perguntas sobre os fatores associados ao parto humanizado e o nível de satisfação relacionadas a algumas assistências prestadas pelos profissionais.

Foram incluídas no estudo puérperas de parto vaginal com idade superior a 18 anos, e a amostra foi composta por 120 puérperas tendo como base o número de partos vaginais realizados na maternidade por mês. As participantes que aceitaram participar do estudo foram instruídas a assinar o Termo de Consentimento Livre e Esclarecido antes da aplicação do formulário.

Para análise estatística os dados coletados foram registrados em um banco de dados de Planilha do Programa Microsoft Excel, posteriormente exportados para análise no software SPSS - Statical Package for the Social Sciences (versão 20). Os resultados foram descritos em tabelas de frequência absoluta e percentual. As associações entre as variáveis foram obtidas pelo teste qui-quadrado onde a significância foi considerada $\mathrm{p}<0,05$ para estabelecer a caracterização por faixa etária e por profissionais.

Esta técnica permite medir o grau de discrepância entre o conjunto de frequências observadas e o conjunto de frequências esperadas, e a partir destes resultados pode-se verificar se os dados observados se ajustam a determinada expectativa. O nível de confiança adotado foi de $95 \%$. As coletas de dados foram iniciadas somente após a submissão e aprovação do projeto pelo Comitê de Ética e Pesquisa da Universidade Federal do Maranhão sob o número do CAAE 18290519.0.0000.5087 em 16 de outubro de 2019. 


\section{Resultados e Discussão}

O Parto Humanizado é uma estratégia que aos poucos vem sendo adotada por diversas redes hospitalares, e visa reduzir o número de cirurgias cesarianas desnecessárias evitando possíveis complicações e contribuindo para um parto mais natural e fisiológico. A pesquisa contou com a participação de 120 puérperas de parto vaginal com idades entre 18 e 41 anos.

O perfil sociodemográfico das participantes mostra que 66,7\% são mulheres de cor parda, 41,7\% têm o ensino médio completo e 49,2\% estão em união estável. Já 72,5\% têm até 2 filhos incluindo o do parto atual e 49,2\% têm uma renda de até 1 salário-mínimo. A assistência humanizada prestada as puérperas foram de grande relevância para esse estudo e mostra que $73,3 \%$ das participantes consideram que o seu parto foi humanizado levando em consideração todos os cuidados prestados na hora do parto.

Os métodos não farmacológicos de alívio da dor foi a principal estratégia usada por parte da equipe de enfermagem para um parto menos doloroso e o mais abordado foram as massagens que eram realizadas durante o trabalho de parto ativo. A autoavaliação sobre o conhecimento do parto humanizado foi o principal instrumento usado para que as participantes fizessem uma autoavaliação quanto ao nível de conhecimento sobre esse tema, que aos poucos é implementado em partos vaginais.

A Tabela 1 mostra a análise da frequência das variáveis (raça, escolaridade, estado civil, $n^{\circ}$ de filhos e renda) associados com a idade. A comparação das variáveis sociodemográficas apresenta resultados altamente significativos para $\mathrm{n}^{\circ}$ de filhos, onde 72,5\% das participantes têm entre 1 e 2 filhos e a maiores partes delas encontram-se na faixa etária de 18 a 24 $\operatorname{anos}(\mathrm{p}=0,001)$.

Quanto ao nível de escolaridade houve significância estatística, e segundo os dados 41,7\% possuem o ensino médio completo, porém dentre as faixas etárias, as participantes que têm entre 25 e 34 anos são a maioria que possuem o ensino médio $(\mathrm{p}=0,013)$.

Com relação ao estado civil 49,2\% informaram está em união estável o que corresponde a maior parte das participantes. Na faixa etária de 18 a 24 anos estão a maior parte de puérperas em união estável ( $n=18 ; 27,3 \%$ ), enquanto, 25\% são solteiras, ou seja, relataram não ter nenhum vínculo com ninguém. 
Tabela 1. Dados sociodemográficos (raça, escolaridade, estado civil, número de filhos e renda, correlacionados com a faixa etária) Imperatriz, MA, Brasil, 2020.

\begin{tabular}{|c|c|c|c|c|c|c|c|c|c|}
\hline & \multicolumn{6}{|c|}{ Faixa etária } & \multirow{2}{*}{\multicolumn{2}{|c|}{-Total }} & \multirow{3}{*}{ p-valor } \\
\hline & \multicolumn{2}{|c|}{$18-24$} & \multicolumn{2}{|c|}{$25-34$} & \multicolumn{2}{|c|}{$35-41$} & & & \\
\hline & $\mathbf{n}$ & $\%$ & $\mathbf{n}$ & $\%$ & $\mathbf{n}$ & $\%$ & $\mathbf{n}$ & $\%$ & \\
\hline Raça & & & & & & & & & $0,646^{\mathrm{a}}$ \\
\hline Branca & 9 & 13,6 & 4 & 9,1 & 2 & 20,0 & 15 & 12,5 & $(4,229)^{*}$ \\
\hline Parda & 44 & 66,7 & 32 & 72,7 & 4 & 40,0 & 80 & 66,7 & \\
\hline Preta & 7 & 10,6 & 4 & 9,1 & 2 & 20,0 & 13 & 10,8 & \\
\hline Amarela & 6 & 9,1 & 4 & 9,1 & 2 & 20,0 & 12 & 10,0 & \\
\hline Escolaridade & & & & & & & & & $\mathbf{0 , 0 1 3}{ }^{\mathrm{a}}$ \\
\hline Fundamental incompleto & 14 & 21,2 & 6 & 13,6 & 1 & 10,0 & 21 & 17,5 & $(22,439)^{*}$ \\
\hline Médio Completo & 24 & 36,4 & 21 & 47,7 & 5 & 50,0 & 50 & 41,7 & \\
\hline Fundamental completo & 5 & 7,6 & 4 & 9,1 & 1 & 10,0 & 10 & 8,3 & \\
\hline Superior incompleto & 0 & 0,0 & 1 & 2,3 & 2 & 20,0 & 3 & 2,5 & \\
\hline Médio incompleto & 22 & 33,3 & 10 & 22,7 & 0 & 0,0 & 32 & 26,7 & \\
\hline Superior completo & 1 & 1,5 & 2 & 4,5 & 1 & 10,0 & 4 & 3,3 & \\
\hline Estado civil & & & & & & & & & $0,143^{\mathrm{a}}$ \\
\hline Solteira & 18 & 27,3 & 10 & 22,7 & 2 & 20,0 & 30 & 25,0 & $(9,586)^{*}$ \\
\hline Casada & 11 & 16,7 & 15 & 34,1 & 3 & 30,0 & 29 & 24,2 & \\
\hline União estável & 36 & 54,5 & 19 & 43,2 & 4 & 40,0 & 59 & 49,2 & \\
\hline$\underline{\text { Separada/Divorciada }}$ & 1 & 1,5 & 0 & 0,0 & 1 & 10,0 & 2 & 1,7 & \\
\hline $\mathrm{N}^{\mathbf{0}}$ de filhos & & & & & & & & & $<0,001^{\mathrm{a}}$ \\
\hline $1-2$ & 58 & 87,9 & 22 & 50,0 & 7 & 70,0 & 87 & 72,5 & $(24,083)^{*}$ \\
\hline $3-4$ & 8 & 12,1 & 17 & 38,6 & 1 & 10,0 & 26 & 21,7 & \\
\hline 5 ou mais & 0 & 0,0 & 5 & 11,4 & 2 & 20,0 & 7 & 5,8 & \\
\hline Renda & & & & & & & & & $0,609^{\mathrm{a}}$ \\
\hline Menos que um salário mínimo & 21 & 31,8 & 8 & 18,2 & 5 & 50,0 & 34 & 28,3 & $(8,617)^{*}$ \\
\hline 1 salário mínimo & 29 & 43,9 & 26 & 59,1 & 4 & 40,0 & 59 & 49,2 & \\
\hline 1,5 salário mínimo & 13 & 19,7 & 7 & 15,9 & 1 & 10,0 & 21 & 17,5 & \\
\hline Até 2 salários mínimos & 2 & 3,0 & 2 & 4,5 & 0 & 0,0 & 4 & 3,3 & \\
\hline Mais de 2 salários mínimos & 1 & 1,5 & 1 & 2,3 & 0 & 0,0 & 2 & 1,7 & \\
\hline
\end{tabular}

Fonte: Autores (2020).

Na Tabela 2 estão dispostos os dados relacionados ao tipo e número de partos, assim como a avaliação assistencial, realizada pelas puérperas que participaram deste estudo. Esta avaliação baseia-se nos cuidados e condutas que normalmente acontece nos partos normais. De acordo com as informações 62,5\% das puérperas já obtiveram outros tipos de partos, e 37,5\% relataram parto vaginal $(\mathrm{p}=0,021)$. Pode-se afirmar que houve uma significância estatística nesses dados, e eles apontam que, a maioria das participantes teve experiência com outros tipos de partos em outras gestações.

Com relação a variável do $\mathrm{n}^{\circ}$ de partos vaginais das participantes ela é altamente significativa, visto que, 36,7\% informaram ter histórico de apenas 1 parto vaginal, enquanto 13,3\% informaram ter histórico de 4 ou mais partos vaginais (p= 0,001). Com essas informações, observa-se que o número de partos normais é baixo e ele acabou não sendo uma alternativa em gestas anteriores.

As participantes deste estudo fizeram uma avaliação quanto ao seu acolhimento na maternidade, e a maior parte julgaram ter recebido um bom acolhimento $(n=52 ; 43,3 \%)$. Já 29,2\% julgaram ter recebido um ótimo acolhimento por parte dos profissionais de saúde, e a maior parte delas encontram-se na faixa etária de 35 a 41 anos o que representa $50 \%$ nessa faixa etária $(n=5 ; 50 \%)$.

Também foi avaliado a satisfação quanto a assistência recebida pelos profissionais durante o trabalho de parto onde 40,3\% julgaram ter sido uma boa assistência e 32,8\% como sendo ótima a assistência oferecida. Dentre as faixas etárias, a de 25 a 34 anos teve uma maior prevalência de julgar a assistência no trabalho de parto como excelente $(n=8 ; 18,2 \%)$. 
Quanto a aplicação de métodos não farmacológicos de alívio da dor 61,7\% das participantes relataram ter sido usado algum método de alívio da dor por parte do profissional e 38,3\% não ter sido usado nenhum método. Na maternidade, os partos normais eram feitos tanto por médicos e enfermeiros obstetras havendo uma significância estatística nessa variável onde descreve que $50 \%$ das puérperas informaram que o parto foi realizado por um médico e 49,2\% realizado por um enfermeiro.

Dentre as faixas etárias, houve uma maior prevalência de partos feitos pelos enfermeiros na faixa de 25 a 34 anos (n= 28; 63,6\%). É válido destacar que o parto de umas das participantes ocorreu na própria residência pelo fato de a ambulância não ter chegado ao local em tempo de transportá-la de volta para a maternidade.

O parto humanizado é um modelo de assistência que restringe a gestante a procedimentos cirúrgicos e condutas desnecessárias priorizando o seu bem-estar e do bebê. Diante dessa afirmação, investigou-se a opinião das puérperas sobre o parto humanizado, constatou-se que $73,3 \%$ consideraram que o seu parto foi humanizado enquanto $26,7 \%$ não considerou. A maior tendência em considerar que teve uma assistência humanizada encontra-se na faixa etária de 35 a 41 anos (n= 8; 80\%).

Tabela 2. Tipos de partos e avaliação da assistência correlacionados com a faixa etária, Imperatriz, MA, Brasil, 2020.

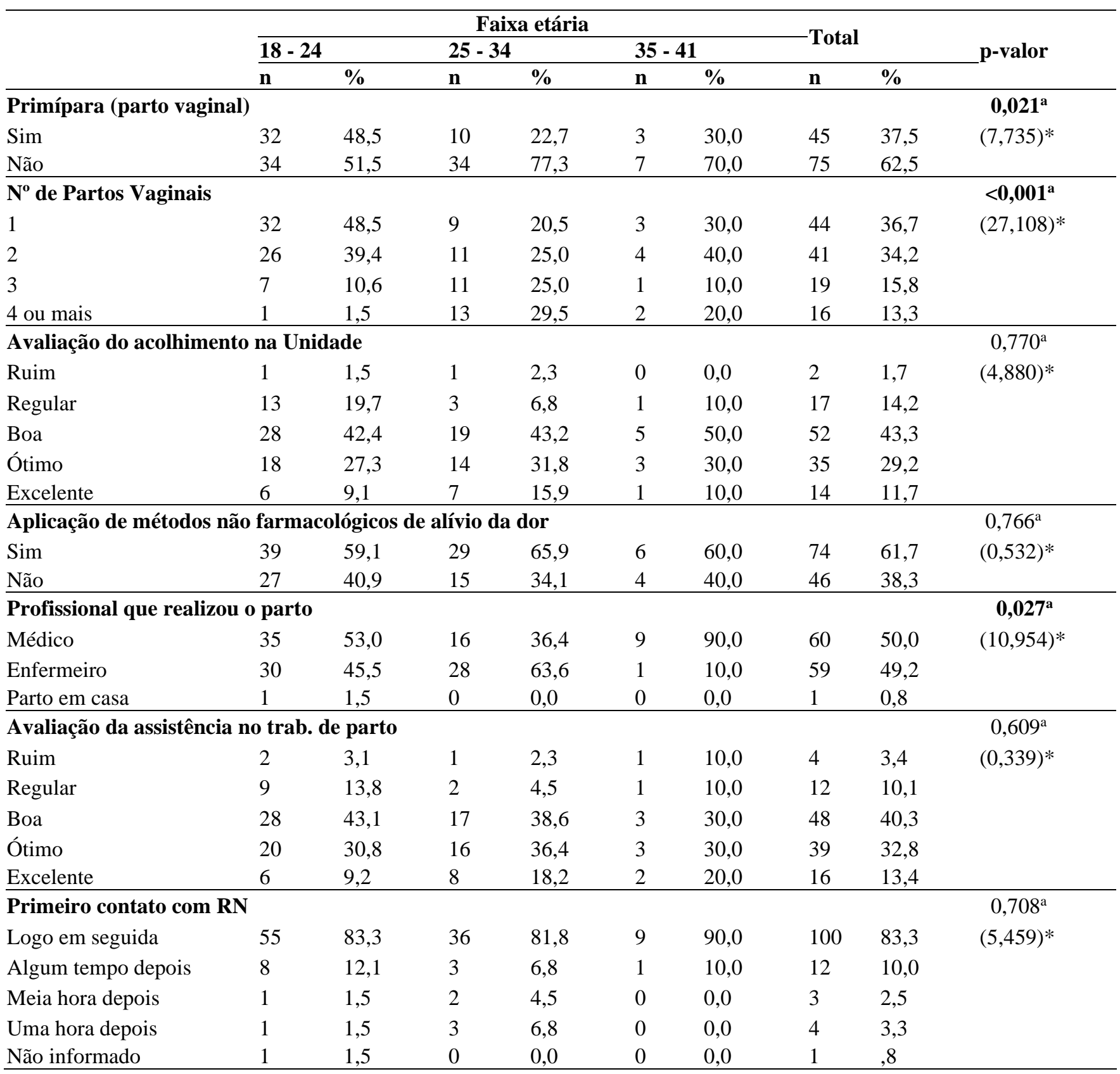


Considera seu parto humanizado

\begin{tabular}{llllllllll} 
Sim & 47 & 71,2 & 33 & 75,0 & 8 & 80,0 & 88 & 73,3 & $(0,442)^{*}$ \\
Não & 19 & 28,8 & 11 & 25,0 & 2 & 20,0 & 32 & 26,7 & \\
\hline
\end{tabular}

Fonte: Autores (2020).

A Tabela 3 mostra os principais métodos não farmacológicos de alívio da dor no parto adotado pelos profissionais da assistência, e de acordo com a Tabela 2, mais de 60\% das mulheres informaram ter sido adotado um ou mais de um método no seu trabalho de parto. O método mais usado foi a deambulação $(n=52 ; 43,3 \%)$, uma vez que, dentro do setor das salas de parto há um corredor onde essas mulheres foram instruídas a deambular com a ajuda do acompanhante. As participantes que informaram terem recebido massagens são $(n=34 ; 28,3 \%)$ e é um método bastante eficaz associado com a deambulação.

Os exercícios respiratórios foi o menos utilizado e de acordo com a pesquisa, 84,2\% relataram não ter sido abordado por parte dos profissionais de enfermagem. A Hidroterapia é a mais eficiente em casos de parto normal, porém em apenas $25,8 \%$ das puérperas ele foi abordado.

Tabela 3. Métodos não farmacológicos utilizados para o alívio da dor no trabalho de parto, Imperatriz, MA, Brasil, 2020.

\begin{tabular}{lcc}
\hline Variáveis & $\mathbf{n}$ & $\mathbf{\%}$ \\
\hline Hidroterapia & 31 & 25,8 \\
Sim & 89 & 74,2 \\
Não & & \\
\hline Deambulação & 52 & 43,3 \\
Sim & 68 & 56,7 \\
Não & & \\
\hline Massagens & 34 & 28,3 \\
Sim & 86 & 71,7 \\
Não & & \\
\hline Exercícios Respiratórios & 19 & 15,8 \\
Sim & 101 & 84,2 \\
Não & & \\
\hline
\end{tabular}

Fonte: Autores (2020).

A Tabela 4 mostra uma autoavaliação do conhecimento das puérperas sobre o parto humanizado. Essa autoavaliação foi feita através da seguinte pergunta: “Qual seu nível de conhecimento sobre Parto Humanizado?”, e as variáveis foram usadas como respostas. No entanto 35\% informaram ter um conhecimento regular sobre o assunto, enquanto 30\% relataram haver um nível conhecimento moderado. Apenas 5,8\% das participantes julgaram ter um nível de conhecimento ótimo sobre esse tipo de parto.

Tabela 4. Autoavaliação sobre o conhecimento relacionado ao Parto Humanizado, Imperatriz, MA, Brasil, 2020.

\begin{tabular}{lll}
\hline Variáveis & $\mathbf{n}$ & \% \\
\hline Insuficiente & 3 & 2,5 \\
Baixo & 32 & 26,7 \\
Regular & 42 & 35,0 \\
Moderado & 36 & 30,0 \\
Ótimo & 7 & 5,8 \\
\hline
\end{tabular}

Fonte: Autores (2020). 


\section{Discussão}

Os resultados desta pesquisa demonstram a adoção de boas práticas assistenciais ao trabalho de parto por meio da visão das puérperas atendidas na maternidade. Constatou-se nesse estudo que o parto humanizado é adotado com efetividade na maternidade, porém durante a realização desta pesquisa pode ser observado que questões estruturais na sala de parto da maternidade não contribuem para a realização de um parto mais humanizado.

Verifica-se com isso, no entanto, é que o ambiente hospitalar precisa de uma reconfiguração constante no campo obstétrico, voltado a perspectiva da humanização do parto (Vargens, Silva e Progianti, 2017). Para que ocorra a implementação da humanização na assistência ao parto, faz-se necessário mais do que conscientização - é necessário mudanças no sistema de saúde e nos profissionais que nela atuam (Mselle, Kohi e Dol, 2018).

As maternidades devem ser projetadas para atender as necessidades específicas da mulher na hora do parto, com uma iluminação adequada, banheiro individual com banheiras grandes com água quente, ventilação adequada e o máximo de privacidade deve ser oferecido (Mascarenhas et al., 2019). A Unidade obstétrica da maternidade encontrava-se sob reforma estrutural, com objetivo de ofertar maior comodidade e conforto para as mulheres, sendo assim, o princípio para novos modelos de atenção a mulher no parto.

A assistência a mulher durante o trabalho de parto requer uma série de cuidados em todos os seus estágios clínicos, isto é, desde o início do trabalho de parto até o período de pós-parto (Ribeiro et al., 2016). A proposta de humanização do parto reconhece a autonomia da mulher como ser humano visando a necessidade de conduzir o momento do parto com práticas que apresentem evidências e permitam aumentar a segurança e bem-estar tanto da mãe como do bebê (Pereira et al., 2016).

Essa estratégia tem a finalidade de estruturar e organizar a atenção à saúde materno-infantil no país e está sendo implementada, gradativamente, em todo território nacional (Brasil, 2020). As práticas para o parto humanizado são realizadas por parte da equipe multiprofissional, sendo considerada uma diretriz do Ministério da Saúde proposta por meio da Rede Cegonha que trabalha com práticas obstétricas sob uma perspectiva mais humanística e segura.

O parto humanizado é dar a todas as mulheres um direito que é garantido a elas com: um atendimento seguro, acolhedor e que respeite suas necessidades físicas, emocionais, psicológicas, sociais e espirituais, independentemente do profissional que esteja executando ou da instituição onde ela se encontra (Silva et al., 2017). A assistência humanizada no parto é definida por vários autores como um resgate do acompanhamento do trabalho de parto e assistência ao parto, respeitando a fisiologia da mulher, oferecendo suporte emocional não apenas para a mulher, como também para família e pessoas que a parturiente escolheu para estarem ao seu lado (Fernandes e Lima, 2016).

Percebe-se que a equipe de enfermagem que atua na maternidade desempenha bem o papel que toda gestante necessita para que possa haver um parto com um olhar mais humanístico, e isso é fundamental que seja executado por todas as instituições de saúde pelo Brasil. A assistência recebida pelas participantes deste estudo, de modo geral, foi boa e contribuiu para dar maior conforto e segurança as mulheres em trabalho de parto.

É importante destacar o contato da mãe com o recém-nascido $(\mathrm{RN})$ que deve ser o mais precocemente possível todas as vezes em que ser oportuno após o nascimento. Esse contato precoce, logo após o nascimento em colocar o RN em contato físico direto com a mãe traz inúmeros benefícios a ambos e fortalece o vínculo afetivo materno-infantil, além de transmitir calor, carinho e conforto.

O primeiro contato pele a pele entre mãe/recém-nascido deve ser iniciado logo após o nascimento sendo contínuo, prolongado e estabelecido entre binômio mãe/recém-nascido (Oliveira et al., 2019). Os primeiros 40 minutos após o nascimento é descrito como fase de inatividade alerta para o bebê e o contato pele a pele deve ser incentivado pela equipe de 
enfermagem visando proporcionar vínculo mãe-filho, aquecer o bebê através do contato pele a pele e estabilizar a frequência cardíaca e respiratória (da Fontoura Machado et al., 2019).

O contato prévio faz parte da assistência humanizada no parto e prevê que os profissionais devam estimular a aproximação entre a mãe e o bebê no pós-parto imediato em contato pele a pele (Alcântara; Malheiros, 2019). No estudo, observou-se que, esse contato prévio ocorreu na maior parte dos partos, independentemente de o parto ter sido realizado por médico ou enfermeiro. O incentivo para que ocorra o contato imediato entre mãe e filho é um procedimento simples e um cuidado que deve ser realizado em todas as unidades obstétricas.

O cuidado no parto humanizado possui vários aspectos positivos que somam e fortalecem o bem-estar das mulheres, tais como: utilização dos métodos não farmacológicos de alívio da dor, presença da doula na sala de parto, um ambiente mais acolhedor e tranquilo dentro das maternidades, a presença e inclusão de um acompanhante masculino na hora do parto, entre outras, são ações indispensáveis de vínculo entre paciente e profissional (Vilela et al., 2019).

A humanização no parto não significa ausência de dor ou que não possa ocorrer intercorrências, e sim dar o direito a mulher de ter o seu filho da forma mais tranquila e confortável, e sem nenhum tipo de violência obstétrica (Silva; Carneiro, 2017). De acordo com as participantes, mais de $70 \%$ consideraram que houve uma humanização no seu parto, em função das condutas adotadas pelos profissionais da Unidade Obstétrica da maternidade como a aplicação dos métodos não farmacológicos de alívio da dor e o acompanhamento durante todo o processo parturitivo pela equipe de enfermagem.

No que diz respeito as ações assistenciais que devem ser executadas pela equipe de enfermagem, destacam-se como principal elo os métodos não farmacológicos de alívio da dor na hora do parto. O banho de aspersão ajuda no relaxamento e controle dos níveis de estresse promovendo menos dores. Em análise com parâmetros neuroendócrinos, seu uso diminui a liberação de cortisol e $\beta$-endorfinas e aumenta a secreção de noradrenalina, fatores ligados ao alívio de condições estressantes (Mascarenhas et al., 2019).

Outros estudos apontam que, o ideal para a mãe e para o filho é ela estar em deambulação durante o trabalho de parto, pois ocorre uma contração uterina e o fluxo sanguíneo que chega até o bebê aumenta, tornando o parto mais rápido e diminuindo a sensação de dor (Camargo et al., 2019). Os exercícios respiratórios agem reduzindo a sensação dolorosa, melhora os níveis de saturação sanguínea materna de $\mathrm{O}^{2}$, proporciona relaxamento e diminui a ansiedade durante o primeiro estágio do trabalho de parto como consequência trazendo maior conforto a mulher (Cruz, 2017).

Já os efeitos da massagem na região cérvice-sacral foram considerados um dos métodos mais eficazes para a redução da dor lombar, principalmente, na primeira fase do trabalho de parto, pois atua reduzindo o estresse, ansiedade e alivia a tensão local, o que ainda permite a participação ativa de um acompanhante (Oliveira et al., 2020). Os métodos não farmacológicos para alívio da dor foram bastante abordados durante o processo parturitivo das participantes, e os mais usados foram a deambulação $(43,3 \%)$ e o uso de massagens $(28,3 \%)$.

Trata-se de práticas de baixo custo e que é facilmente oferecido pelas instituições de saúde, uma vez que, apresentam grande impacto na qualidade da assistência, principalmente por substituírem, na medida do possível o uso de medicações anestésicas e analgésicas (Medeiros et al., 2015). Todos os métodos estão profundamente comprometidos com as políticas de humanização que proporcionam as mulheres a diminuição do medo, satisfação e alívio da dor (Dias et al., 2018). Em todos os casos eram usados pelo menos dois métodos, além de contar algumas vezes com a presença uma fisioterapeuta que auxiliava com os exercícios respiratórios.

Em relação ao grau de conhecimento sobre o parto humanizado pelas participantes, por se tratar de um assunto ainda pouco explorado para elas, de acordo com a visão delas a maior parte tem um certo nível de conhecimento sobre esse assunto, porém, elas atrelam o parto humanizado a um tipo de parto com ausência de dor, como é o caso de um parto cesáreo, o que difere da realidade prática. Essa percepção é influenciada pela falta de conhecimentos e até mesmo ausência de diálogo com os 
profissionais de saúde que as acompanham durante o pré-natal, como também pelo não esclarecimento das dúvidas em relação ao momento e ao tipo de parto (Feitosa et al., 2017).

A comunicação e informação em saúde entre profissional e gestantes precisam ser priorizadas durante o pré-natal, pois é a melhor forma de promover a compreensão do processo gestacional (Apolinário et al., 2016). Por isso, faz-se necessário durante as consultas de pré-natal estabelecer diálogos educativos como forma de preparo a mulher para a maternidade, visto que, tal medida influência sobre a escolha da via de parto pela gestante (dos Santos et al., 2019).

O termo "Humanização" é pouco conhecido pelas puérperas da maternidade, sobretudo atrelado ao parto. Para elas, humanização no parto significa ausência de dor e um olhar mais diferenciado pela equipe de enfermagem, onde o cuidado venha promover atenção, conforto e segurança para a mulher. A maioria das mulheres veem a maternidade como um momento especial na vida, e ao entregar-se, esperam cuidados da equipe de saúde para o seu parto e que as atenções sejam voltadas para ela e o nascimento do bebê, almejando uma assistência humanizada (Ribeiro Filho et al., 2016).

As mulheres que se sentem abandonadas ou que são atendidas por uma equipe que não oferece atenção e cuidados tem a maior possibilidade de possuir percepções negativas quanto ao parto (Álvares et al., 2018). A humanização requer da equipe de enfermagem grande atuação em respeito aos aspectos fisiológicos da parturiente, sem haver intervenções desnecessárias e grande suporte psicoemocional a mulher e aos familiares (Marins et al., 2020). Um bom acolhimento é algo importante a ser feito, pois é um momento em que há bastante medo e insegurança pelas mulheres e na percepção delas, esse acolhimento foi ao encontro com aquilo que elas esperavam receber.

As principais limitações encontradas para realização dessa pesquisa foram os baixos níveis de conhecimento sobre o parto humanizado e a insegurança de algumas parturientes em participar da pesquisa, apesar de todos os esclarecimentos terem sido dados, algumas restringiram-se a não participar, além disso, a Unidade Obstétrica encontrava-se em reforma estrutural, o que provocou dificuldades na aplicação da pesquisa. É notório que as salas de parto não eram adequadas e não oferecia a estrutura ideal para um parto humanizado e isso de certo modo refletiu negativamente na percepção das puérperas. Percebe-se através desse estudo que é possível promover um parto mais humanizado por meio de ações assistenciais executadas pela equipe de enfermagem durante todo o processo parturitivo.

Os dados revelam que essa proposta de "humanização" ainda é pouco conhecida pelas mulheres, e a ausência de diálogo por parte dos profissionais pode trazer conceitos equivocados por parte das mulheres. Por isso durante o pré-natal é importante que a mulher seja orientada quanto ao momento do parto, e conheça os benefícios do parto normal em todos os casos em que ele seja viável acontecer. Isso evita que certos equívocos quanto a adoção do parto normal e fica claro a importância da adoção do parto humanizado.

\section{Conclusão}

Levando-se em conta o que foi observado, a assistência no parto humanizado é algo extremamente fundamental no cuidado a mulher em processo parturitivo. Ficou claro o quanto as práticas assistenciais realizadas pela equipe de enfermagem e mostradas nesse estudo são princípios para a adoção do parto humanizado, e são técnicas baseadas em evidências científicas de grande eficácia, o que proporciona a mulher um parto mais seguro e humano contribuindo para o bem-estar maternoinfantil.

A equipe de enfermagem são os principais idealizadores desse processo, em que durante todos os momentos ofereceram todo o suporte necessário para as parturientes. Para isso, faz-se necessário que os profissionais de enfermagem ofereçam um cuidado com um olhar mais acolhedor para com as mulheres. Além disso, esse estudo incentivou o interesse das participantes a respeito da humanização no parto e esclareceu diversos equívocos quanto a esse modelo de atenção. 
Nesse cenário, o enfermeiro é o principal profissional capaz de dar autonomia e protagonismo a mulher durante o processo parturitivo mediante estratégias que venham esclarecer dúvidas e acima de tudo o preparo da mulher para o momento do parto fazendo com que o momento venha ser vivenciado de forma positiva e especial em suas vidas.

Diante do exposto é de suma importância o desenvolvimento de novas pesquisas, com delineamento voltado à assistência ao parto humanizado, onde sejam abordados a assistência de enfermagem, salientando a importância das técnicas com evidências científicas, utilizadas no cuidado humanizado.

\section{Referências}

Alcântara, L. M.; Malheiros, I. S.; Reis, M. A. D. (2020). Parto Humanizado: primeiro contato entre mãe e filho após o nascimento. http://repositorio.aee.edu.br/bitstream/aee/8520/1/TCC\%20ISAURA\%20SILVA\%20\%20MALHEIROS\%20\%20E\%20LUIZA\%20MIGUEL\%20ALC\%c3\%8 2NTARA.pdf.

Alvares, A. S., Corrêa, Á. C. D. P., Nakagawa, J. T. T., Teixeira, R. C., Nicolini, A. B., \& Medeiros, R. M. K. (2018). Práticas humanizadas da enfermeira obstétrica: contribuições no bem-estar materno. Revista Brasileira de Enfermagem, 71, 2620-2627. DOI: http://dx.doi.org/10.1590/0034-7167-2017-0290.

Apolinário, D., Rabelo, M., Wolf, L. D. G., Souza, S. R. R. K., \& Leal, G. C. G. (2016). Práticas na atenção ao parto e nascimento sob a perspectiva das puérperas. Rev Rene, 17(1), 20-28.

Brasil. Ministério da Saúde. Rede Cegonha. (2020). https://www.saude.gov.br/acoes-e-programas/rede-cegonha/sobre-o-programa.

Brasil. Ministério da Saúde. Secretaria Executiva. (2002). Programa Humanização no Parto: Humanização no Pré-natal e Nascimento. <http://bvsms.saude.gov.br/bvs/publicacoes/parto.pdf>.

Camargo, C. M; Vaz, L. G.; Oliveira, A. S.; Costa, C. S. (2019). A eficácia dos métodos não farmacológicos aplicados pelo enfermeiro obstetra no alívio da dor do trabalho de parto. Rev Cient Esc Est Saúde Pública Goiás “Cândido Santiago”. 5(2):64-75.

Cruz, M. O.; Manobras para o alívio da dor durante o trabalho de Parto Normal. https://bvsms.saude.gov.br/bvs/publicacoes/diretrizes_nacionais_assistencia_parto_normal.pdf.

Dias, E. G., Ferreira, A. R. M., Martins, A. M. C., Nunes, M. M. D. J., \& Alves, J. C. S. (2018). Eficiência de métodos não farmacológicos para alívio da dor no trabalho de parto normal. Enferm. foco (Brasília), 35-39.

dos Santos, A. B. B., de Melo, E. V., de Gonçalves Dias, J. M., da Nóbrega Didou, R., de Araujo, R. A. S., de Oliveira Santos, W., \& Araújo, L. B. (2019). Grau de conhecimento das gestantes do serviço público sobre parto humanizado. ABCS Health Sciences, 44(3).

Estrela, C. (2018). Metodologia Científica: Ciência, Ensino, Pesquisa. Editora Artes Médicas.

Feitosa, R. M. M., Pereira, R. D., de Paula Souza, T. J. C., de Freitas, R. J. M., Cabral, S. A. R., \& de França Souza, L. F. (2017). Fatores que influenciam a escolha do tipo de parto na percepção das puérperas. Revista de Pesquisa Cuidado é Fundamental Online, 9(3), 717-726.

Fernandes, N.; Lima, C. Humanização na assistência de enfermagem no parto natural. (2016). http://temasemsaude.com/wpcontent/uploads/2016/09/16307.pdf

Fontoura Machado, C., Gehlen, M. H., de Souza, M. H. T., \& dos Santos, N. O. (2019). Cuidado de enfermagem na promoção do contato pele a pele mãe-filho na primeira hora de vida. Disciplinarum Scientia Saúde, 20(2), 485-496.

Marian, D. C., Barros, A. G. D. S., \& Flach, J. K. (2018). Evolução temporal dos partos cesáreos e dos fatores maternos associados no Brasil no período de 2000 a 2015. Medicina-Pedra Branca.

Marins, R. B., Cecagno, S., Gonçalves, K. D., Braga, L. R., Ribeiro, J. P., \& Soares, M. C. (2020). Care techniques for pain relief in birthing/Tecnologias de cuidado para o alívio da dor na parturição. Revista de Pesquisa: Cuidado é Fundamental Online, 12, $276-281$.

Mascarenhas, V. H. A., Lima, T. R., Silva, F. M. D., Negreiros, F. D. S., Santos, J. D. M., Moura, M. Á. P., ... \& Jorge, H. M. F. (2019). Evidências científicas sobre métodos não farmacológicos para alívio a dor do parto. Acta Paulista de Enfermagem, 32(3), 350-357.

Medeiros, J., Hamad, G. B. N. Z., Costa, R. R. D. O., Chaves, A. E. P., \& Medeiros, S. M. D. (2015). Métodos não farmacológicos no alívio da dor de parto: percepção de puérperas. Espaç Saúde [Internet], 16(2), 37-44.

Mselle, L. T., Kohi, T. W., \& Dol, J. (2018). Barriers and facilitators to humanizing birth care in Tanzania: findings from semi-structured interviews with midwives and obstetricians. Reproductive health, 15(1), 1-10.

Oliveira, B. S. D., Batista, S. G., Valcarenghi, R. V., Mattos, A. R. D. S., Correia, J. B. B., \& Hoffmann, A. C. O. D. S. (2019). Contato precoce pele a pele entre mãe e recém-nascido: contribuições da enfermagem em uma maternidade de São José/SC. Revista Eletrônica Estácio Saúde, 9(1), 8-16.

Oliveira, L. S., De Oliveira, L. K. P., Rezende, N. C. C. G., Pereira, T. L., \& Abed, R. A. (2020). Uso de medidas não farmacológicas para alívio da dor no trabalho de parto normal. Brazilian Journal of Health Review, 3(2), 2850-2869. 
Research, Society and Development, v. 10, n. 6, e18310615451, 2021

(CC BY 4.0) | ISSN 2525-3409 | DOI: http://dx.doi.org/10.33448/rsd-v10i6.15451

Pereira, S. S., dos Santos Oliveira, I. C. M., da Silva Santos, J. B., \& Carvalho, M. C. D. M. P. (2016). Parto natural: a atuação do enfermeiro diante da assistência humanizada. Tempus Actas de Saúde Coletiva, 10(3), ág-199.

Possati, A. B., Prates, L. A., Cremonese, L., Scarton, J., Alves, C. N., \& Ressel, L. B. (2017). Humanização do parto: significados e percepções de enfermeiras. Escola Anna Nery Revista de Enfermagem, 21(4), 1-6. doi: 10.1590/2177-9465-ean-2016-0366, 2017.

Ribeiro Filho, J. F., Machado, P. H. F., da Silva Araújo, K. R., \& de Abreu Sepúlvedra, B. (2016). Assistência ao parto normal sob o olhar da parturiente. Revista Eletrônica Gestão e Saúde, (1), 113-125.

Siabani, S., Jamshidi, K., \& Mohammadi, M. M. (2019). Attitude of pregnant women towards Normal delivery and factors driving use of caesarian section in Iran (2016). BioPsychoSocial medicine, 13(1), 8.

Silva, E. M., \& Carneiro, P. A. P. (2017). A importância da humanização da assistência de enfermagem ao parto normal. http://192.100.247.84/handle/prefix/319.

Sousa, A. M. M., Souza, K. V. D., Rezende, E. M., Martins, E. F., Campos, D., \& Lansky, S. (2016). Práticas na assistência ao parto em maternidades com inserção de enfermeiras obstétricas, em Belo Horizonte, Minas Gerais. Escola Anna Nery, 20(2), 324-331.

Vilela, A. T., Tenório, D. D. S., Silva, R. M. D. S., Silva, J. C. B. D., \& Albuquerque, N. L. A. (2019). Percepção dos enfermeiros obstetras diante do parto humanizado. Rev. enferm. UFPE on line, 1-6.

Vargens, O. M. D. C., Silva, A. C. V. D., \& Progianti, J. M. (2017). Contribuição de enfermeiras obstétricas para consolidação do parto humanizado em maternidades no Rio de Janeiro-Brasil. Escola Anna Nery, 21(1). 